\title{
Normality and Disease
}

\author{
Donald B. Calne and Joanna S. Calne
}

\begin{abstract}
There have been trends to equate normal with an ideal state of health, and disease with disturbances that are determined solely by subclinical abnormalities. While in any living language there is a conflict between established definition and the need for change, modification in the use of words that are of such central importance to medical writing requires cogent justification that has not been forthcoming in these instances. To avoid further obscuration of the literature, the term normal should be limited to its traditional connotation of average, and the term disease should be reserved for disturbances of health that are clinically manifest.
\end{abstract}

RÉSUMÉ: L'état normal et la maladie. L'état normal a souvent été défini comme un état de santé idéal et la maladie comme des désordres qui sont déterminés exclusivement par des anomalies subcliniques. Même si dans toute langue vivante il y a un conflit entre la définition établie et le besoin de changement, des modifications dans l'usage des mots qui ont une importance capitale dans la littérature médicale demande une justification valable qui ne s'est pas manifestée dans le cas présent. Pour éviter une plus grande confusion dans la littérature, le terme normal devrait être limité à sa connotation traditionnelle de moyen et le terme maladie devrait être réservé aux désordres de la santé qui se manifestent cliniquement.

Can. J. Neurol. Sci. 1988; 15:3-4

Among the most frequently misused words in current medical literature are the terms normal and disease. Before attempting to analyze the problems we shall illustrate them by examining the difficulties that stem from the varied inferences associated with normal and disease.

In the case of normal, a good example is the debate over whether cerebral glucose metabolism decreases with aging. If we take normal to mean average, then we must conclude that cerebral glucose metabolism declines in the elderly, because the mean value for an unselected population is lower at the age of 70 years than at the age of 20 years.

However, there is a current trend to force the meaning of normal in another direction. By excluding the many elderly subjects who inevitably see less, hear less, remember less and move less than they did at the age of 20 years, some investigators present an ideal minority as normal. From this latter definition, we would conclude that cerebral glucose metabolism does not change with age. In this example, the same observations lead to opposite conclusions, according to how we define normality. The importance of the issue deserves emphasis, because the very notion of a pathological or clinical entity depends upon a firmly based concept of what is normal.
The meaning of disease is similarly confused. A group of clinical features "running together" constitutes a syndrome, for which there may be many causes. In contrast, any definition of disease includes some consideration of specific etiology. Thus headache is a symptom; headache with neck stiffness is a syndrome (meningismus); headache with neck stiffness and meningococci in the cerebrospinal fluid is a disease - (meningococcal meningitis). Hemiplegia is not a disease because we recognize a variety of causes. Where, then, do we stand with Parkinson's disease? A range of agents, such as toxins, drugs, and viruses, may lead to Parkinsonism (tremor, rigidity and bradykinesia) but what of the patients with these clinical features but no identifiable specific etiology? How we define disease determines how we interpret pathological findings such as Lewy bodies. These eosinophilic intraneural inclusions occur abundantly in patients with idiopathic Parkinsonism and much less frequently in elderly subjects without symptoms. Are Lewy bodies a subclinical marker for a disease, or a histological feature of old age?

We can ask this question in simpler and more striking terms - how do we identify illness in the elderly? Is old age a syndrome? Without a coherent definition of normal and disease 
there is no answer. With appropriate definitions, it is clear that the elderly experience declining health as a normal experience, without necessarily encountering disease.

Having discussed the problems arising from the current usage of normal and disease, we shall now consider definitions that may help resolve the confusion.

\section{Normality}

A reasonable first step in the interpretation of language, is to refer to the dictionary. The Oxford English Dictionary ${ }^{1}$ defines normal as usual and there is no connotation of ideal. How then, has the concept of medical normality become saddled with the implication of supreme good health? The answer is, at least in part, wish-fulfillment. We live in a society with $9 \%$ of the population over 64 years of age, compared with $5 \%$ in $1921^{2}$ and a predicted $20 \%$ in $2031 .{ }^{3}$ We strive to deny the inevitable deterioration which precludes our enjoying the same quality of life in old age, that we experience in youth.

The debility associated with old age may be exacerbated by individual diseases. A pertinent question is why do these diseases occur more frequently late in life? We may not know the answer to this, but we must not mislead ourselves by selecting an ideal control group to represent the elderly in clinical studies.

A reasonable concept of normality can be formulated in statistical terms. Modern technology provides an array of increasingly sensitive diagnostic tests for measuring biological chemistry, structure and function. These allow asymptomatic variables such as blood biochemistry to be recorded over a vast demographic range of subjects. Tails in the statistical distribution curve can be identified with ease. In this setting, the issue of defining normality becomes easier. Normal can be construed as a range of findings falling within two standard errors of the mean for a given population at a given time (taking into account such variables as age and sex). What of those who lie outside the arbitrary statistical limits? They are, by definition, abnormal. We must now decide whether they have a disease.

\section{Disease}

Statistical outliers for any physiological, biochemical or anatomical measurement cannot be regarded as necessarily having a disease. Short stature is no more a disease then exceptionally good eye sight. What, then, constitutes a disease? The Oxford English Dictionary ${ }^{1}$ defines disease as "illness" or "sickness". The origin of the word disease is relevant; it clearly implies a disturbance of ease, from which it is reasonable to infer the presence of symptoms.

However, a disease is more than a collection of symptoms, as illustrated in the previous discussion of headache and neck stiffness. A disease is a constellation of clinical features deriving from a distinct cause, and running a characteristic course in space (anatomy) and time (natural history). Difficulties arise where etiology has not been identified. Here it is generally assumed that the clinicopathological entity derives from a single cause. This theoretical construct, the clinicopathological entity and the assumed single cause, frequently acquires the status of a disease.
Is the patient suffering from a disease when he or she is asymptomatic, but investigation reveals a malignant growth, an abnormal gene, a high blood sugar or antibodies to a virus such as HIV? While there are varying degrees of likelihood that these circumstances produce clinical features, it is not practicable to base a definition upon an inaccessible probability value. Many abnormalities associated with disease are clinically latent. One in a thousand subjects with antibodies to polio virus manifest the symptoms of poliomyelitis; a far smaller proportion with varicella antibodies develop herpes zoster; encephalitis is a distinct rarity among those with vaccinia antibodies. From these considerations it seems inescapable that to sustain epistemological consistency a disease must be associated with clinical disturbances. Physiologists, biochemists and pathologists may determine abnormalities and measure deleterious processes, but clinicians define diseases. In so doing, correlations may be established between clinical features and abnormal investigations. Subclinical disturbances may play an important role in sharpening the precision of a clinical diagnosis and of course it is mandatory, where possible, to treat latent abnormalities that are known to have a high probability of leading to clinical deficits (as in the case of asymptomatic malignant growth).

The relationship between Parkinson's disease and Lewy bodies, already mentioned, exemplifies the problem. Lewy bodies are found in both Parkinsonian patients and elderly asymptomatic subjects. Pathologists increasingly describe the presence of Lewy bodies without symptoms as evidence of "Lewy body disease" but who can say which individuals with abnormal biochemical, physiological or morphological findings will develop sickness, illness or disease?

Alzheimer's disease poses a similar problem. Here it is inferred that deteriorating memory and cognitive function have been investigated to exclude such causes as cerebral tumour or hypothyroidism, and the physician is left with an idiopathic group that is presumed to have a single but unknown cause. However, there are no compelling reasons for expecting that there is only one cause. Pathology has been invoked to help in the designation of Alzheimer's dementia, but cerebral morphology is not usually available during life, and the histological markers seldom offer adequate specificity. Neurofibrillary tangles and senile placques are abundant in Alzheimer's dementia, but they also occur in normal senescence. The situation is analogous to that already discussed for Lewy bodies. While we recognize the need for flexibility in language, excessive license with such fundamental terms as normal and disease corrupts meaning and courts confusion.

\section{REFERENCES}

1. The Oxford English Dictionary. Oxford University Press, Oxford. 1981.

2. Sixth Census of Canada. Volume 2. Department of Trade and Commerce, Ottawa. 1921.

3. Abelson J, Paddon P, Strohmenger C. Perspectives on Health. In: Statistics Canada. Catalogue 82-540E, Ministry of Supply and Services Canada. Ottawa. 1983. 